\title{
An Atypical Presentation of Mycobacterium Avium Complex Pneumonia
}

\author{
Tobe Momah* \\ Department of Family Medicine, University of Mississippi Medical Center, USA
}

*Corresponding author: Tobe Momah, Department of Family Medicine, University of Mississippi Medical Center, USA, Tel: 1-6019846800; Fax: 1-6019845420; E-mail: tmomah@umc.edu; tobemomah@yahoo.com

Received: 27 Jan, 2018 | Accepted: 18 Jan, 2019 | Published: 25 Jan, 2019

Citation: Momah T (2019) An Atypical Presentation of Mycobacterium Avium Complex Pneumonia. J HIV AIDS 5(1): dx.doi. org/10.16966/2380-5536.162

Copyright: (C) 2019 Momah T. This is an open-access article distributed under the terms of the Creative Commons Attribution License, which permits unrestricted use, distribution, and reproduction in any medium, provided the original author and source are credited.

\section{Introduction}

48 year old African American Male patient with PMHx of HIV and Bell's palsy presented to the Emergency Room of a community hospital with high grade fever $\left(102^{\circ} \mathrm{f}\right)$, cough, progressive loss of weight (20 pounds in under one month) and chest pain. He stated that he had been told sixteen years ago that he had HIV but was not started on treatment at the time. Patient denied any hemoptysis but endorses his chest pain as worse on deep inspiration, graded as 7/10 and associated with progressively worsening Shortness of Breath (SOB), Dyspnea on Examination (DOE) and increased sweating for nearly a month. Patient's PCP had done a chest-X ray which, according to patient, showed "spots on his lungs." Prior to presentation in the hospital, patient had taken two seven-day courses of levofloxacin without any improvement.

On evaluation in the Emergency Room (ER), a Computed Tomography Angiography (CTA) Chest was ordered to rule out pulmonary embolism. It showed nodular pulmonary opacities, with moderate-sized left pleural effusion, splenic lesions and axillary/ mediastinal lymphadenopathy (Figure 1). His laboratory results were significant for CD4 count of 214 , HIV viral load of 48,700, anemia (Hemoglobin/Hematocrit-9.8/29.6), increased total protein $(8.9 \mathrm{mg} / \mathrm{dl})$ and decreased albumin $(2.9 \mathrm{gm} / \mathrm{dl})$. Otherwise, his other routine laboratory values were normal. Patient was placed in respiratory isolation pending sputum Acid-Fast Bacilli (AFB) smear and culture results. Meanwhile, his quantiferon gold assay returned negative, and after consultation with Infectious Diseases (ID) and pulmonary specialists, patient was started on Zithromax, Piperacillintazobactam, and vancomycin.

In spite of these antibiotics, patient kept spiking fevers. He also became progressively more dyspneic and, as a result, underwent an ultrasound guided thoracentesis to relieve the massive left sided pleural effusion. $1500 \mathrm{ml}$ of exudative fluid was withdrawn, and assayed. The pleural fluid cytology, as well as his blood cultures, was negative for malignancy or infection respectively. Two weeks after admission, patient's respiratory culture on solid media returned positive for Mycobacterium Avium Complex (Figure 2). Prior to that, patient had undergone a left sided tube thoracostomy to drain recurrent pleural effusion and a bronchoscopy, axillary lymph node biopsy and bone marrow aspiration for diagnostic purposes. Candida Albicans, seen in the bronchus on bronchoscopy, were the only significant findings.

Patient was thereafter started on oral clarithromycin, rifampin and Ethambutol. He subsequently became afebrile, and was discharged to follow-up with the ID and pulmonary physicians. He began his HAART medication, as an outpatient, as well as continued treatment for M.A.C (which will last a total of twenty-four months). Since discharge, patient has had a repeat CT Chest with contrast (Figure 3) which showed improved nodular consolidations in the right upper lobe. Right lower lobe lesions were, however, slightly more prominent suggesting progression of disease in those areas while left pleural effusion was small and loculated.

\section{Discussion and Conclusion}

Mycobacterium Avium Complex (MAC) is a hard to diagnose respiratory condition commonly seen in immunocompromised HIV patients with AIDS. It consists of two Mycobacteria species that include Mycobacterium intracellulare and Mycobacterium Avium. These Non-Tuberculous Mycobacterial (NTM) infections are one of the most common infections seen in patients infected with HIV, and in patients suffering with AIDS showed an increased threefold mortality before the advent of HAART [1].

The incidence of MAC is increasing all the more as the survival of profoundly immunocompromised patients is prolonged. There are unknown factors as regards the precise origin of these infections and as to the exact epidemiology of atypical mycobacterial [2]. It has, however, been found in multiple sites including water, soil, and animals in less than $15 \%$ of cases [3]. MAC is also not transmitted via aerosol or contact between humans or from animals to humans [3].

In a recent study done on MAC patient in Portugal, out of the 25 patients with NTM disease, 23(92\%) had pulmonary disease (either localized or disseminated) but only 13(56\%) fulfilled the current ATS/ IDSA criteria for pulmonary NTM disease (Table 1). Our patient had pulmonary involvement, as do majority of our patients, but it took 
Table 1: Clinical and Microbiologic Criteria for Diagnosing Nontuberculous Mycobacterial (NTM) Lung Disease.

\begin{tabular}{|c|c|}
\hline \multicolumn{2}{|r|}{ Clinical (both required) } \\
\hline 1. & $\begin{array}{l}\text { Pulmonary symptoms, nodular or cavitary opacities on chest radiograph, or a high-resolution computed tomography scan that shows } \\
\text { multifocal bronchiectasis with multiple small nodules }(A, I)^{*} \\
\text { (and) }\end{array}$ \\
\hline 2. & Appropriate exclusion of other diagnoses $(A, I)$ \\
\hline \multicolumn{2}{|r|}{ Microbiologic } \\
\hline 1. & $\begin{array}{l}\text { Positive culture results from at least two separate expectorated sputum samples (A,II). If the results from (1) are non-diagnostic, consider } \\
\text { repeat sputum AFB smears and cultures (C,III) (Or) }\end{array}$ \\
\hline 2. & Positive culture result from at least one bronchial wash or lavage $(\mathrm{C}, \mathrm{III})(\mathrm{Or})$ \\
\hline 3. & $\begin{array}{l}\text { Transbronchial or other lung biopsy with mycobacterial histopathologic features (granulomatous inflammation or AFB) and positive culture } \\
\text { for NTM or biopsy showing mycobacterial histopathologic features (granulomatous inflammation or AFB) and one or more sputum or } \\
\text { bronchial washings that are culture positive for } \operatorname{NTM~}(A, I)\end{array}$ \\
\hline 4. & $\begin{array}{l}\text { Expert consultation should be obtained when NTM are recovered that are either infrequently encountered or that usually represent } \\
\text { environmental contamination (C,III) }\end{array}$ \\
\hline 5. & $\begin{array}{l}\text { Patients who are suspected of having NTM lung disease but do not meet the diagnostic criteria should be followed until the diagnosis is } \\
\text { firmly established or excluded (C,III) }\end{array}$ \\
\hline 6. & $\begin{array}{l}\text { Making the diagnosis of NTM lung disease does not, per se, necessitate the institution of therapy, which is a decision based on potential } \\
\text { risks and benefits of therapy for individual patients (C,III) }\end{array}$ \\
\hline
\end{tabular}

*Strength recommendations based on quality of evidence (adapted from the Infectious Disease Society of America/United States) Public Health Service Rating System.

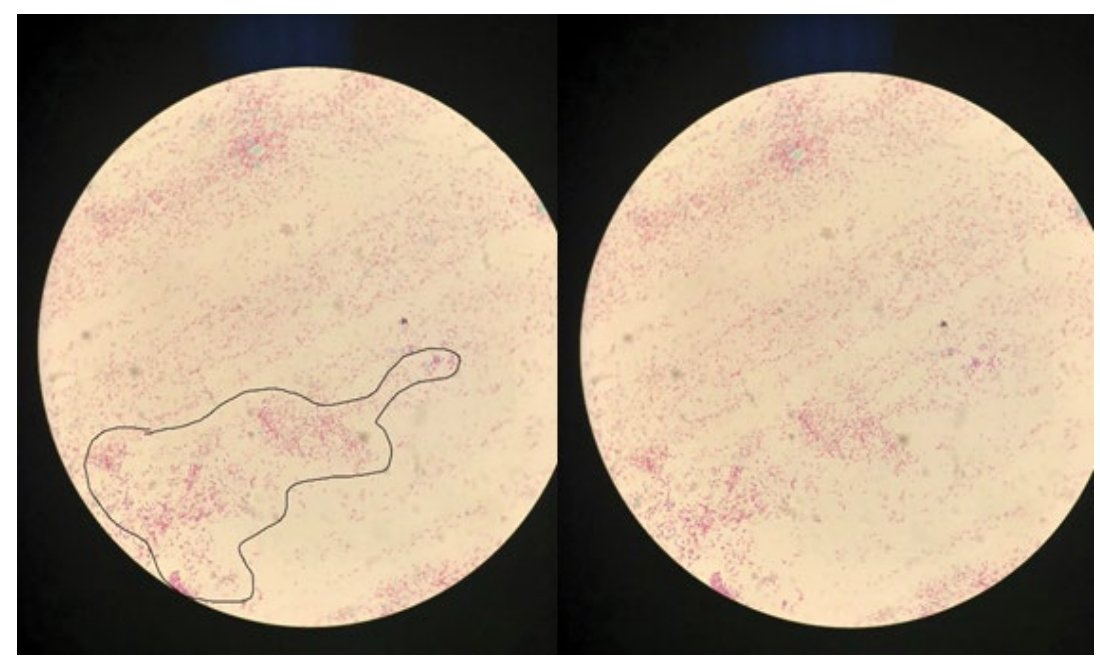

Figure 1: Chest $\mathrm{X}$ ray with bilateral pleural effusion.

sixteen days to make a diagnosis in his case due to difficulty isolating his culture. Most cases in the scientific literature are diagnosed through blood or sputum cultures4, but in the case of our patient these tests were repeatedly negative.

A delay in accurate diagnosis of NTM has been shown to lead to an increased mortality, especially in immunocompromised patients. The medication employed may also have significant side effects that can lead to treatment withdrawal and an increased resistance to the first-line drugs, and less than $50 \%$ of these drugs are successful, and relapses are estimated at $>50 \%$ within 3 year [4]. The need to expedite diagnosis in MAC, therefore, cannot be overemphasized.
The reason for accuracy in diagnosis of MAC is non-the-more emphasized than in its treatment. With high toxicity, and a low invivo efficacy, the main line treatment modalities for MAC including Clarithromycin, Azithromycin, Ethambutol and Rifabutin, are used for a minimum of twelve months in a triple therapy format. When these are not well tolerated or contra-indicated, an addition of streptomycin, levofloxacin or rifampin to the regimen may be indicated.

The resistance to treatment, notwithstanding, recurrence seems to be an even bigger problem in the treatment of MAC. In a recent metaanalysis, examining the long-term efficacy of macrolide containing antibiotics, the patients who grew MAC in their sputum within one 


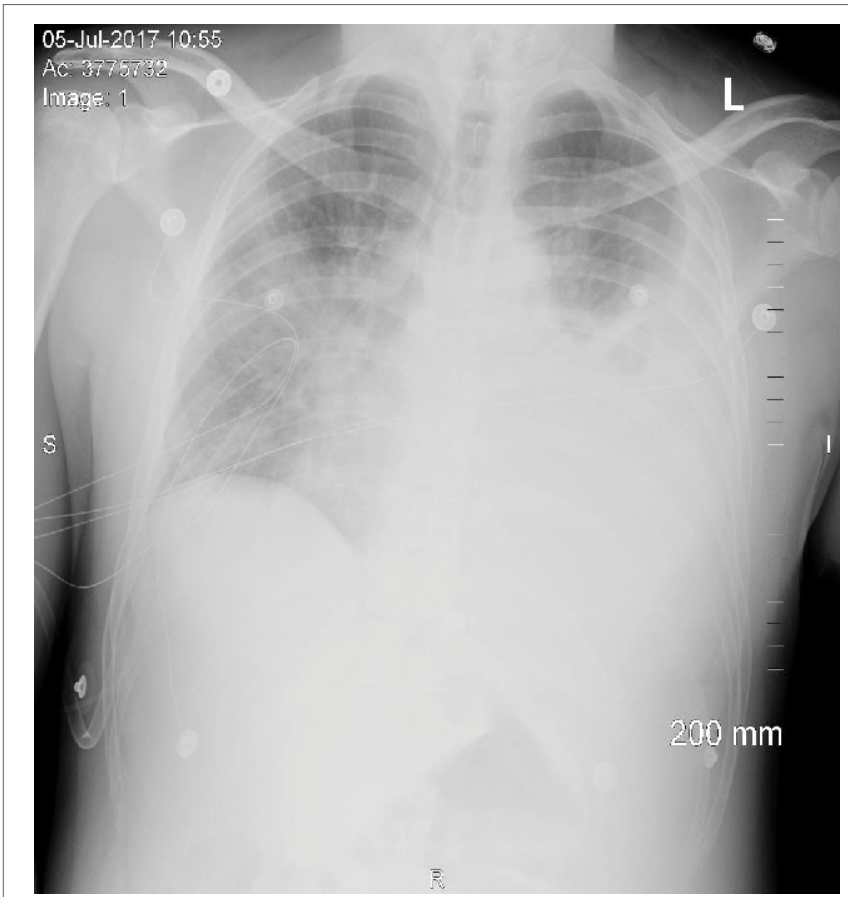

Figure 2: Respiratory culture showing Mycobacterium Avium Complex.

year of discontinuing an eighteen-month treatment regimen, were $32 \%$ of the total number of patients treated $[5,6]$. In our patient, the ID physicians elected to do a twenty-four month treatment regimen in order to avoid recurrence.

\section{References}

1. Chaisson RE, Gallant JE, Keruly JC, Moore RD (1998) Impact of opportunistic disease on survival in patients with HIV infection. AIDS 12: 29-33.

2. Payen MC, De Wit S, Clumeck N (1997) Manifestations, diagnosis and treatment of non-tuberculous mycobacterial infections in patients with HIV infection. Rev Mal Respir 14: S142-S151.

3. Griffith DE, Aksamit T, Brown-Elliott BA, Catanzaro A, Daley C, et al. (2007) An official ATS/IDSA statement: Diagnosis, treatment, and

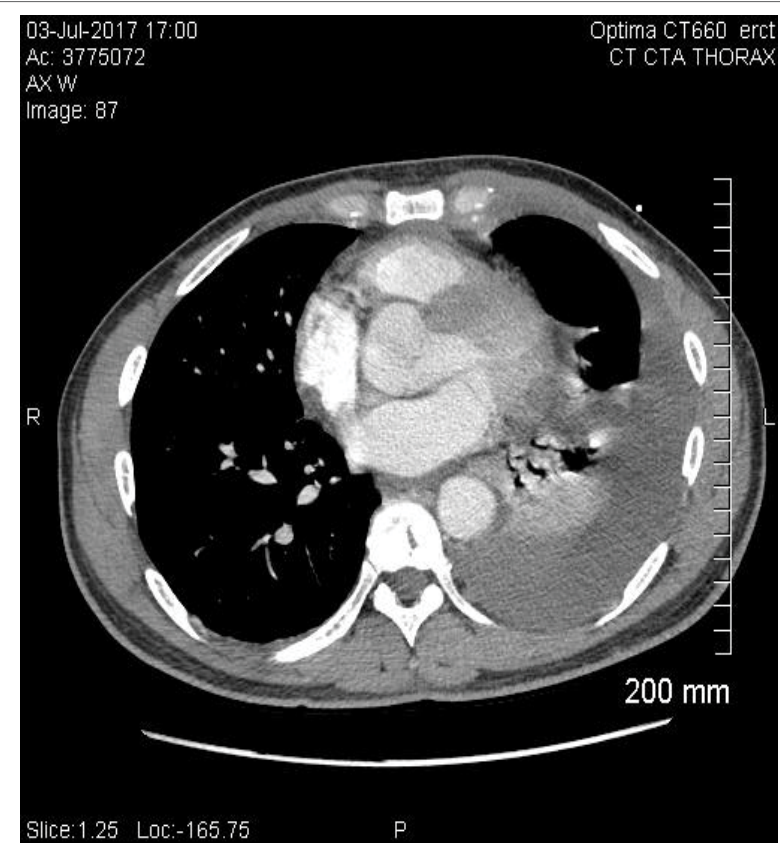

Figure 3: CT Chest showing improved left pleural effusion but with persistent loculation.

prevention of Nontuberculous mycobacterial diseases. Am J Respir Crit Care Med 175: 367-416.

4. Huang HL, Cheng MH, Lu PL, Shu CC, Wang JY, et al. (2017) Epidemiology and Predictors of NTM Pulmonary Infection in Taiwan-a Retrospective, Five-Year Multicenter Study. Sci Rep 7: 16300.

5. Hernandez-Solís A, Cicero-Sabido R, González-Villa M, MartínezRivera II, Mandujano-Martínez ADP, et al. (2017) Nontuberculous mycobacteria in clinical samples with negative acid-fast bacilli. Int J Mycobacteriol 6: 391-395.

6. Hasegawa N, Nishimura T, Ohtani S, Takeshita K, Fukunaga K, et al. (2009) Therapeutic effects of various initial combinations of chemotherapy including clarithromycin against Mycobacterium avium complex pulmonary disease. Chest 136: 1569-1575. 